\title{
Heart Rate Variability Analysis Guided by Respiration in Major Depressive Disorder
}

\author{
Spyridon Kontaxis ${ }^{1}$, Michele Orini ${ }^{2}$, Eduardo Gil ${ }^{1,3}$, Mar Posadas-de Miguel ${ }^{4}$, Maria Luisa Bernal ${ }^{4}$, \\ Jordi Aguiló $^{3,5}$, Concepción de la Camara ${ }^{4,6}$, Pablo Laguna ${ }^{1,3}$, Raquel Bailón ${ }^{1,3}$ \\ ${ }^{1}$ BSICoS Group, I3A, IIS Aragón, University of Zaragoza, Spain \\ ${ }^{2}$ Mechanical Engineering Department and Barts Heart Centre at St Bartholomews Hospital, UK \\ ${ }^{3}$ CIBER de Bioingeniería, Biomateriales y Nanomedicina, Spain \\ ${ }^{4}$ Instituto de Investigación Sanitaria de Aragón (IIS Aragón), Zaragoza, Spain \\ ${ }^{5}$ Microelectronics and Electronic Systems Dept., Autonomous University of Barcelona, Spain \\ ${ }^{6}$ CIBER de Salud Mental, Spain
}

\begin{abstract}
In this study a Heart Rate Variability (HRV) analysis guided by respiration to evaluate different patterns of $\mathrm{Au}$ tonomic Nervous System (ANS) in response to a cognitive stressor between Major Depressive Disorder (MDD) and control (CT) subjects is presented. Cardiorespiratory Time Frequency Coherence (TFC) reveals the local coupling of $H R V$ and respiration signal which is essential and usually not included in estimation of ANS measures derived by HRV. Parasympathetic activity of ANS is measured as the power at the frequencies where TFC between HRV and respiration is significant, whereas sympathetic dominance is measured as the normalized power in the low frequency band [0.04, 0.15] Hz of HRV excluding the power of those frequencies related to respiration. Results showed significantly lower $(p<0.05)$ sympathetic dominance in MDD with respect to CT subjects during stress, suggesting that ANS reactivity as response to stress stimuli is lower in MDD patients. The study of ANS reactivity to a stressor may serve as a biomarker useful for the early diagnosis and monitoring of MDD patients.
\end{abstract}

\section{Introduction}

Major Depressive Disorder (MDD) is the most prevalent psychiatric disease, which was ranked as the third leading cause of global disability in the world [1]. MDD has been linked with an autonomic imbalance (increased sympathetic tone), which has been projected as one of the underlying mechanisms of various diseases [2].

Heart Rate Variability (HRV) has been widely used for the noninvasive assessment of Autonomic Nervous System (ANS). Reduced HRV has been reported in MDD com- pared to control subjects, suggesting a shift of autonomic balance toward sympathetic activation [3], while contradictory results have been reported in [4].

A possible explanation for the lack of consensus regarding the potential use of HRV may be the comorbidity of mental disorders. In particular, comorbid anxiety disorder is one of the strongest predictors of MDD severity [5]. Both depression and anxiety are associated with cognitive deficits and changes in brain function. As a consequence, ANS changes induced by depression may blur ANS changes induced by anxiety. Another possible reason for the above inconsistencies, beyond the heterogeneity of the applied methods, is the estimation of HRV indices. In the frequency domain, HRV has been subdivided into a high frequency band (HF, $[0.15,0.4] \mathrm{Hz})$, whose power reflects the parasympathetic activity, and a low frequency band (LF, $[0.04,0.15] \mathrm{Hz}$ ), reflecting both sympathetic and parasympathetic ANS activity. However, respiration affects HRV through changes in respiratory frequency and respiratory pattern and thus, analysis of a simultaneously recorded respiration signal should be considered when analyzing HRV [6]. Furthermore, in [7] it has been found that HRV analysis guided by respiration improved the ability of HRV to discriminate cognitive stress in healthy subjects. To the best of our knowledge, respiratory information has seldom been taken into account by former HRV approaches in MDD [8,9], which could lead to erroneous interpretation of HRV indices.

In this approach a time-frequency (TF) coherence analysis between HRV and respiration to define the TF regions where the signals are locally coupled is adopted $[10,11]$. The joint analysis of respiration and HRV is conducted not only to reveal those frequencies related to parasympathetic activity (respiration) along time, but also to identify overlaps occurring between components at frequencies with 
different physiological meaning, e.g. LF and HF. Furthermore, the natural reactivity of ANS in MDD in response to arousal changes is paramount and only few studies have investigated this aspect [9]. In the present study, ANS response will be evaluated first in MDD patients and controls during a basal condition, then during a cognitive stress task (cardiovascular reactivity) and finally during a recovery condition.

\section{Materials and Methods}

\subsection{Experimental Protocol}

A database of 11 MDD patients ( 6 women, age $45.36 \pm$ 13.04 years, Body Mass Index (BMI) $24.51 \pm 2.65$ $K g / \mathrm{m}^{2}$ ), recruited from consultation as well as from the psychiatric inpatient ward, and 11 controls, abbreviated as CT, (matched by sex, BMI, age) was recorded at the Hospital Clínico Lozano Blesa (Zaragoza). MDD group consists of subjects with clinically significant depression who were evaluated based on the Diagnostic and Statistical Manual of Mental Disorders (DSM-5) and the Hamilton Rating Scale for Depression (HRSD > 19). The recordings comprise the following stages:

i. Basal condition $\left(C_{\mathrm{B}}\right)$, where the subjects are filling psychometric tests; in particular, perceived stress scale (PSS), State-Trait Anxiety Inventory test (STAI), Stress Visual Analogue Scale (SVAS), Scale of Stress Symptoms (ES3), Beck Depression Inventory (BDI).

ii. Stress condition $\left(C_{\mathrm{S}}\right)$, where the subjects are performing the Trail Making Test (TMT).

iii. Recovery condition $\left(C_{\mathrm{R}}\right)$, where the subjects requested to relax during 5 minutes and are supposed to return to a basal or resting stage.

Respiratory signal (thoracic belt) as well as 3 orthogonal ECG leads were continuously recorded using Medicom system (ABP10 module of Medicom MTD, Ltd, Russia) at a sampling frequency $\left(f_{s}\right)$ of 250 and $1000 \mathrm{~Hz}$, respectively.

\subsection{HRV analysis guided by respiration}

Beat occurrence time series were automatically detected on lead $Y$ and HRV signal was generated applying on those the integral pulse frequency modulation model, which accounts for the presence of ectopic beats [12], and subsequently was corrected by the mean heart rate [13]. Both $\mathrm{HRV}$ and respiration were resampled at $f_{s}=4 \mathrm{~Hz}$ and band-pass filtered in the range $[0.04,0.8] \mathrm{Hz}$. The coupling between HRV $(x(t))$ and respiration $(y(t))$ is partially related with the parasympathetic control of the heart and lungs. The respiratory influences on HRV is an everchanging phenomenon which can be captured based on TF coherence $\hat{\gamma}(t, f)$ (TFC) by means of Smoothed Pseudo
Wigner Ville Distribution (SPWVD) [10]:

$$
\hat{\gamma}(t, f)=\frac{\left|\hat{S}_{\mathrm{XY}}(t, f)\right|}{\sqrt{\hat{S}_{\mathrm{XX}}(t, f) \hat{S}_{\mathrm{YY}}(t, f)}}
$$

where $\hat{S}_{\mathrm{XX}}(t, f), \hat{S}_{\mathrm{YY}}(t, f), \hat{S}_{\mathrm{XY}}(t, f)$ are power spectral densities defined based on SPWVD:

$$
\begin{aligned}
\hat{S}_{\mathrm{XY}}(t, f) & =\mathcal{F}_{(v, \tau) \rightarrow(t, f)}\left\{A_{\mathrm{XY}}(v, \tau) \Phi(v, \tau)\right\} \\
A_{\mathrm{XY}}(v, \tau) & =\mathcal{F}_{t \rightarrow v}\left\{x\left(t+\frac{\tau}{2}\right) y^{*}\left(t-\frac{\tau}{2}\right)\right\} \\
\Phi(v, \tau) & =\exp \left\{-\pi\left[\left(\frac{v}{v_{0}}\right)^{2}+\left(\frac{\tau}{\tau_{0}}\right)^{2}\right]^{2 \lambda}\right\}
\end{aligned}
$$

where $A_{\mathrm{XY}}(v, \tau)$ is the cross-ambiguity function of signals $x(t)$ and $y(t), \Phi(v, \tau)$ a simplified version of the multiform-tiltable exponential kernel $\left(v_{0}=0.045, \tau_{0}=\right.$ $0.05, \lambda=0.3$ ) to suppress the interference terms and $\mathcal{F}$ the Fourier transform operator, used to pass from the ambiguity function domain $(v, \tau)$ to the TF domain $(t, f)$.

Estimation of TF regions, characterized by a significant coherence level, is established based on a surrogate data analysis. To do that, $x(t)$ and $y(t)$ are defined as two white gaussian noises and a TFC statistical threshold $\left(\gamma_{\mathrm{TH}}(t, f)\right)$ is constructed based on the distribution of coherence values after 250 repetitions of those uncorrelated processes with $1 \%$ risk of concluding that $x(t)$ and $y(t)$ are coupled when there is no actual coupling (null hypothesis).

Regions with statistically significant coupling $(\hat{\gamma}(t, f) \geq$ $\left.\gamma_{\mathrm{TH}}(t, f)\right)$ are identified by a TF mask $M(t, f)$ of logical values, where 0 and 1 mean lack and presence of coupling, accordingly. The portions of $M(t, f)$ that are smaller than a rectangle of sides $2 \mathrm{~s} \mathrm{x} \Delta_{f} / 2 \mathrm{~Hz}$ are removed, with $\Delta_{f}$ being a term related to the frequency resolution (see [11]), adding robustness to the final estimates. The set of time instants where respiration and HRV signal were coupled $\left(t \in \Omega_{\mathrm{C}}\right)$ and when those coupling regions were overlapping with $\mathrm{LF}$ band $\left(t \in \Omega_{\mathrm{C}}^{\mathrm{LF}}\right)$ for a bandwidth percentage higher than $25 \%$, i.e. $0.0275 \mathrm{~Hz}$, can be easily derived through $M(t, f)$. The subset of time instants where HRV indices will be measured is $t \in \Omega_{\mathrm{M}} \equiv\left\{t \in \Omega_{\mathrm{C}} ; t \notin \Omega_{\mathrm{C}}^{\mathrm{LF}}\right\}$.

Parasympathetic activity which, in this study refers to all respiratory-driven components in HRV, is measured by:

$$
P_{\mathrm{R}}(t)=\int_{0.04}^{f_{\mathrm{HR}}(t) / 2} \hat{S}_{\mathrm{Xx}}(t, f) M(t, f) d f, \forall t \in \Omega_{\mathrm{M}}
$$

where no estimation is conducted during overlapping $(t \in$ $\Omega_{\mathrm{C}}^{\mathrm{LF}}$ ) and the maximum analyzed frequency at each time instant is half the instantaneous heart rate $\left(f_{\mathrm{HR}}(t)\right)$. Sympathetic activity is measured in LF band as:

$$
P_{\mathrm{L}}(t)=\int_{0.04}^{0.15} \hat{S}_{\mathrm{Xx}}(t, f)(1-M(t, f)) d f, \forall t \in \Omega_{\mathrm{M}}
$$



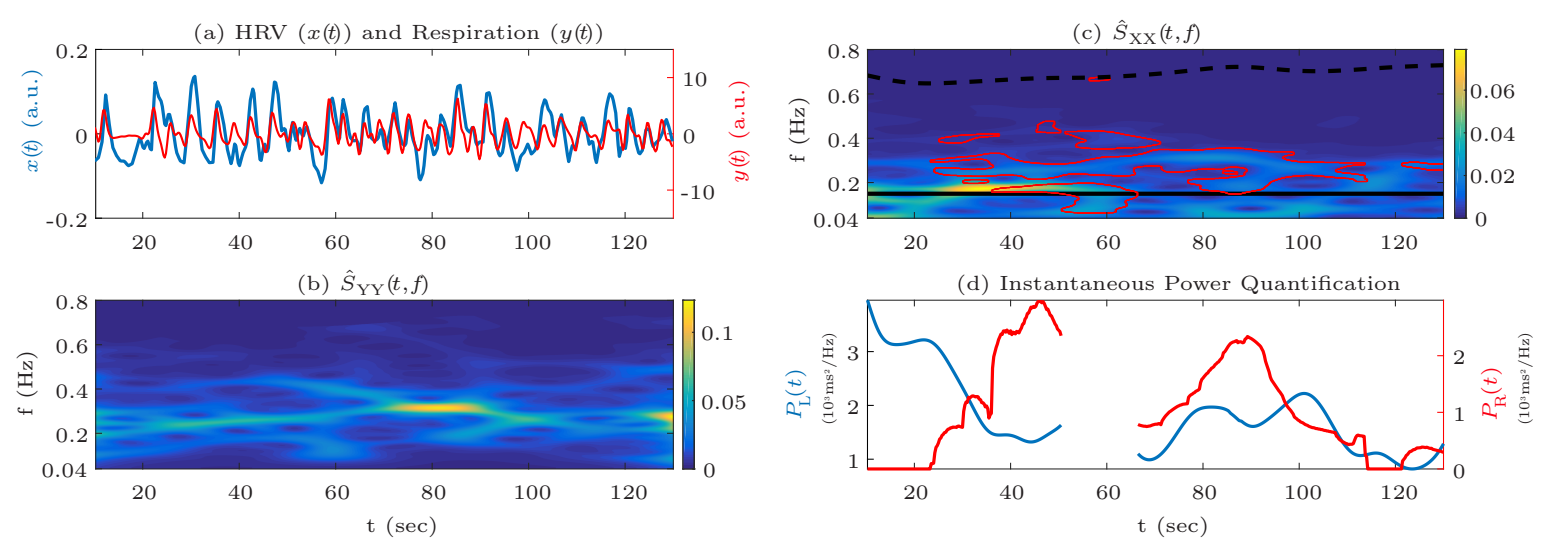

Figure 1. (a) An example of HRV $(x(t))$ and Respiration $(y(t))$ signals in $C_{\mathrm{B}}$, (b) SPWVD of $y(t)$, (c) SPWVD of $x(t)$ where in red are marked the areas with significant coupling $\left(\mathcal{T}_{\mathrm{C}}=73.6 \%\right)$ defined by the TF mask $M(t, f)$. Black line denotes the upper limit of LF band, i.e. $0.15 \mathrm{~Hz}$, while the dashed line $f_{\mathrm{HR}}(t) / 2$ (d) Instantaneous power for LF band $\left(P_{\mathrm{L}}(t)\right)$ and respiratory-related components $\left(P_{\mathrm{R}}(t)\right)$. Estimation is conducted $\left(\mathcal{T}_{\mathrm{M}}=62.3 \%\right)$ only in absence of overlapping between the areas defined by $M(t, f)$ and $\mathrm{LF}$ band $\left(\mathcal{T}_{\mathrm{C}}^{\mathrm{LF}}=11.3 \%\right)$.

where those frequencies related to respiration are excluded through the term $(1-M(t, f))$ (Fig. 1). Instantaneous ratio (7) and normalized ratio (8), which are related to sympathovagal balance, are defined as:

$$
\begin{array}{lll}
R_{\mathrm{L} / \mathrm{R}}(t) & =P_{\mathrm{L}}(t) / P_{\mathrm{R}}(t), & \forall t \in \Omega_{\mathrm{M}} \\
R_{\mathrm{L} / \mathbb{R}}^{\mathrm{N}}(t) & =P_{\mathrm{L}}(t) /\left(P_{\mathrm{L}}(t)+P_{\mathrm{R}}(t)\right), & \forall t \in \Omega_{\mathrm{M}}
\end{array}
$$

The time percentages where $t \in \Omega_{\mathrm{C}}, t \in \Omega_{\mathrm{C}}^{\mathrm{LF}}, t \in \Omega_{\mathrm{M}}$ are abbreviated as $\mathcal{T}_{\mathrm{C}}, \mathcal{T}_{\mathrm{C}}^{\mathrm{LF}}, \mathcal{T}_{\mathrm{M}}$, respectively.

\section{3. $\quad$ Statistical Analysis}

Feature set consists of the temporal median values of the above mentioned parameters i.e. $\bar{P}_{\mathrm{R}}, \bar{P}_{\mathrm{L}}, \bar{R}_{\mathrm{L} / \mathrm{R}}$ and $\bar{R}_{\mathrm{L} / \mathrm{R}}^{\mathrm{N}}$ and the time percentage $\mathcal{T}_{\mathrm{C}}$ where respiration and HRV were coupled. An unpaired statistical test between MDD and CT group is conducted to examine if ANS response is different in a specific condition, while a paired test could reveal significant ANS changes within the same subject at different conditions. Student t-tests or Wilcoxon tests were implemented for each feature depending on the data distribution (Lilliefors test), Gaussian or not, respectively.

\section{Results for ANS related indices}

In three subjects subintervals ( $1 \mathrm{MDD}$ at $C_{\mathrm{B}}$ and 2 $\mathrm{CT}$ at $\left.C_{\mathrm{R}}\right), \mathcal{T}_{\mathrm{C}}^{\mathrm{LF}}$ was higher than $60 \%$, and so were excluded from further analysis. $\mathcal{T}_{\mathrm{C}}^{\mathrm{LF}}$ (median \pm interquartile range) in CT group, at subintervals $C_{\mathrm{B}}, C_{\mathrm{S}}, C_{\mathrm{R}}$ were $13.6 \pm 20.4 \%, 23.9 \pm 22.1 \%, 25.7 \pm 26.1 \%$, respectively, and in MDD group were $22.4 \pm 11.1 \%, 15.2 \pm 18.9 \%$, $10.5 \pm 34.2 \%$, respectively. $\mathcal{T}_{\mathrm{M}}$ in CT group at $C_{\mathrm{B}}, C_{\mathrm{S}}, C_{\mathrm{R}}$ were $64.36 \pm 28.9 \%, 28.4 \pm 27.9 \%, 29.8 \pm 38.7 \%$, respec- tively, and in MDD group were $54.7 \pm 36.0 \%, 56.3 \pm 22.9 \%$, $59.4 \pm 38.5 \%$, respectively.

Results of statistical analysis for ANS related indices derived by HRV in CT and MDD patients are shown in Fig. 2. Statistical differences $(p<0.05)$ between MDD and CT group are observed during $C_{\mathrm{S}}$ for the ratios $\bar{R}_{\mathrm{LR}}$, $\bar{R}_{\mathrm{L} / \mathrm{R}}^{\mathrm{N}}$ and during $C_{\mathrm{R}}$ for the ratio $\bar{R}_{\mathrm{L} / \mathrm{R}}$. In CT subjects ratios $\bar{R}_{\mathrm{L} / \mathrm{R}}$ and $\bar{R}_{\mathrm{L} / \mathrm{R}}^{\mathrm{N}}$ are significantly higher during $C_{\mathrm{S}}$ than during $C_{\mathrm{B}}$, while $\mathcal{T}_{\mathrm{C}}$ is lower. In MDD patients no significant differences were found in $\bar{R}_{\mathrm{L} / \mathrm{R}}$ and $\bar{R}_{\mathrm{L} / \mathrm{R}}^{\mathrm{N}}$ between stress and basal stages, although a significant decrease was observed during recovery with respect to stress.

\section{Discussion}

In the present study a new method for instantaneous tracking of ANS changes has been conducted by means of time-frequency coherence which allows the quantification of sympathetic and parasympathetic activity through HRV signal using respiratory information, and also accounts for exclusion of time instants where components at frequencies with different physiological meaning are overlapping.

Differences in ANS related indices derived from HRV between depressed (MDD) and control (CT) subjects before, during and after a cognitive stressor have been explored. Regarding CT group and its ANS response to the stress task $\left(C_{\mathrm{S}}\right)$, the reduced time percentage $\mathcal{T}_{\mathrm{C}}$ (Fig. 2.e) with respect to the basal condition $\left(C_{\mathrm{B}}\right)$ implies a reduction in the coupling between HRV and respiration that could be due to a vagal withdraw which is shown also by an increment of $\bar{R}_{\mathrm{L} / \mathrm{R}}$ and $\bar{R}_{\mathrm{L} / \mathrm{R}}^{\mathrm{N}}$ ratio (Fig. 2.c-d). On the contrary, MDD group presented significant changes in ratios with respect to post-task relaxation and not compared to basal state, which can be due to the small size of the database. 

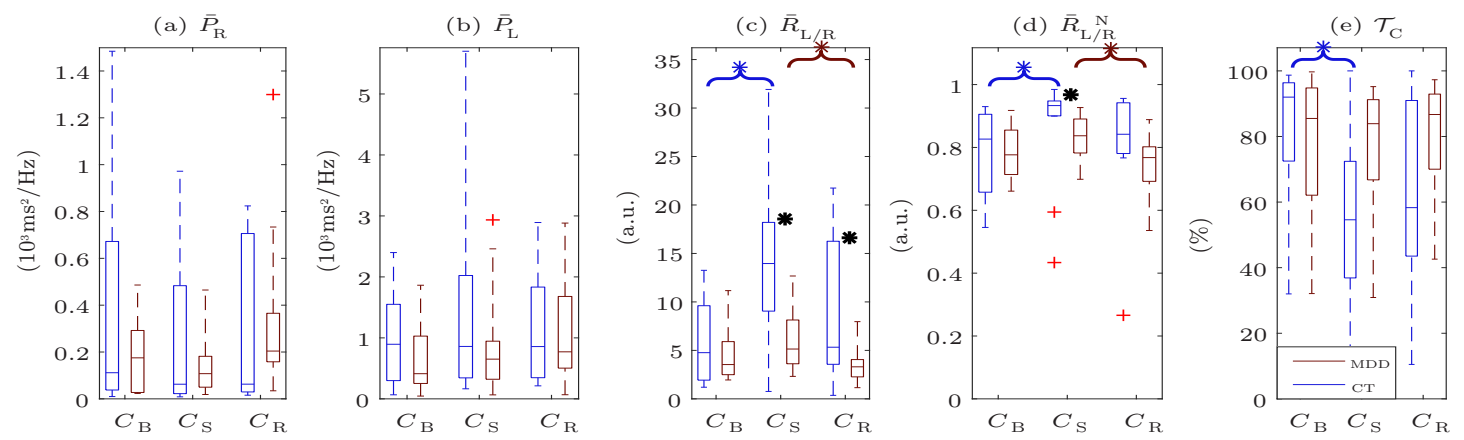

Figure 2. Boxplots of feature values (a) $\bar{P}_{\mathrm{R}}$, (b) $\bar{P}_{\mathrm{L}}$, (c) $\bar{R}_{\mathrm{LR}}$, (d) $\bar{R}_{\mathrm{LR}}^{\mathrm{N}}$, (e) $\mathcal{T}_{\mathrm{c}}$, used in statistical analysis for CT (Blue) and MDD (Red) subjects in each condition $\left(C_{\mathrm{B}}, C_{\mathrm{S}}, C_{\mathrm{R}}\right)$. Black asterisks correspond to statistically significant differences $(p<0.05)$ found in the unpaired analysis between groups at a specific condition, while the colored ones are referring to paired analysis within the same subject at different conditions.

A comparison of $\mathrm{HRV}$ indices showed an ANS passiveness in MDD patients who seemed to be less affected by the ANS stimulation than the healthy subjects, fact that is shown by lower $\bar{R}_{\mathrm{LR}}, \bar{R}_{\mathrm{LR}}^{\mathrm{N}}$ in MDD than CT group. The lower performance of MDD in TMT measured as the time required for accomplishing the task $110.0 \pm 50.2 \mathrm{sec}$ (median \pm interquartile range) in comparison with $\mathrm{CT}$ subjects $66.0 \pm 27.5 \mathrm{sec}$ is in accordance with the above results, suggesting a lower autonomic reactivity in MDD subjects.

Analogous HRV findings on depression were reported in $[8,9]$, where parasympathetic branch was found to be less inhibited during a task condition in MDD patients, who probably are not capable to adjust their mental state to abrupt behavioral changes. Statistical differences in $\bar{R}_{\mathrm{LR}}$ and not in $\bar{R}_{\mathrm{LR}}^{\mathrm{N}}$ during relaxation could be due to the sensibility of unnormalized ratio to outliers presence rather than to possible effects of task execution on the subsequent rest condition. In contrast to previous studies, no significant differences during basal conditions between $\mathrm{CT}$ and MDD patients were observed, which may be due to the different methodology used here, since HRV is corrected by the mean heart rate, or can be due to the reduced sample size.

\section{Conclusion}

Heart rate variability analysis guided by respiration during a cognitive stress revealed significantly decreased ANS reactivity to stress stimuli in MDD patients with respect to matched controls. This information might be useful for the early diagnosis and monitoring of MDD patients.

\section{Acknowledgment}

This work was supported by Ministerio de Economía y Competitividad under the project TIN2014-53567-R, Aragón Government with a personal grant to S. Kontaxis, European Social Fund (EU) through BSICoS group (T96) and CIBER in Bioengineering, Biomaterials \& Nanomedicine through Instituto de Salud Carlos III. Authors acknowledge Marisa Bellido for her support in data collection.

\section{References}

[1] Vos T, et al. Global, regional, and national incidence, prevalence, and years lived with disability for 310 diseases and injuries, 19902015: a systematic analysis for the global burden of disease study 2015. The Lancet 2016;388(10053):1545-1602.

[2] Cuijpers P, et al. Comprehensive meta-analysis of excess mortality in depression in the general community versus patients with specific illnesses. Am J Psychiatry 2014;171(4):453-462.

[3] Wang Y, et al. Altered cardiac autonomic nervous function in depression. BMC Psychiatry 2013;13(1):187.

[4] Kemp AH, et al. Impact of depression and antidepressant treatment on heart rate variability: a review and meta-analysis. Biol Psychiatry 2010;67(11):1067-1074.

[5] Zhiguo W, Yiru F. Comorbidity of depressive and anxiety disorders: challenges in diagnosis and assessment. Shanghai Arch Psychiatry 2014;26(4):227.

[6] Bernardi L, et al. Effects of controlled breathing, mental activity and mental stress with or without verbalization on heart rate variability. J Am Coll Cardiol 2000;35(6):1462-1469.

[7] Hernando A, et al. Inclusion of respiratory frequency information in heart rate variability analysis for stress assessment. IEEE J Biomed Inform 2016;20(4):1016-1025.

[8] Shinba T, et al. Decrease in heart rate variability response to task is related to anxiety and depressiveness in normal subjects. Psychiatry Clin Neurosci 2008;62(5):603-609.

[9] Shinba T. Altered autonomic activity and reactivity in depression revealed by heart-rate variability measurement during rest and task conditions. Psychiatry Clin Neurosci 2014;68(3):225-233.

[10] Orini M, et al. Characterization of dynamic interactions between cardiovascular signals by time-frequency coherence. IEEE Trans Biomed Eng 2012;59(3):663-673.

[11] Orini M, et al. Assessment of the dynamic interactions between heart rate and arterial pressure by the cross time-frequency analysis. Physiol Meas 2012;33(3):315.

[12] Mateo J, Laguna P. Analysis of heart rate variability in the presence of ectopic beats using the heart timing signal. IEEE Trans Biomed Eng 2003;50(3):334-343.

[13] Bailón R, et al. The integral pulse frequency modulation model with time-varying threshold: application to heart rate variability analysis during exercise stress testing. IEEE Trans Biomed Eng 2011;58(3):642-652.

Address for correspondence:

Spyridon Kontaxis (sikontax@unizar.es)

Campus Río Ebro, C/ María de Luna 1, 50018 Zaragoza, Spain 